\title{
«L'année Baudelaire» 22, textes recueillis par J. Dupont et A. Schellino
}

\section{Ida Merello}

\section{(2) OpenEdition}

1 Journals

\section{Edizione digitale}

URL: https://journals.openedition.org/studifrancesi/21491

DOI: 10.4000/studifrancesi.21491

ISSN: 2421-5856

\section{Editore}

Rosenberg \& Sellier

\section{Edizione cartacea}

Data di pubblicazione: 1 décembre 2019

Paginazione: 593-594

ISSN: 0039-2944

\section{Notizia bibliografica digitale}

Ida Merello, ««L'année Baudelaire» 22, textes recueillis par J. Dupont et A. Schellino», Studi Francesi [Online], 189 (LXIII | III) | 2019, online dal 01 mars 2020, consultato il 11 novembre 2021. URL: http:// journals.openedition.org/studifrancesi/21491 ; DOI: https://doi.org/10.4000/studifrancesi.21491

Questo documento è stato generato automaticamente il 11 novembre 2021.

\section{(c) (i) (9)}

Studi Francesi è distribuita con Licenza Creative Commons Attribuzione - Non commerciale - Non opere derivate 4.0 Internazionale. 


\title{
«L'année Baudelaire» 22, textes recueillis par J. Dupont et A. Schellino
}

\author{
Ida Merello
}

\section{NOTIZIA}

«L'année Baudelaire» 22, textes recueillis par J. Dupont et A. Schellino, Paris, Champion, 2018, $244 \mathrm{pp}$.

1 Questo numero dell'«Année Baudelaire» costituisce un omaggio a Claude Pichois e proprio per questo è organizzato come una raccolta di note erudite: trentasei interventi, dall'impostazione rigorosamente filologica: si tenta qui di render conto della maggior parte, nell'impossibilità di elencarli tutti. Nel suo insieme questa «Année» permette una maggiore definizione dei rapporti di Baudelaire con $i$ suoi contemporanei, grazie a oggetti ritrovati - foto, lettere, ritratti, incisioni -, di chiarire meglio i suoi interventi giornalistici o il significato che B. attribuisce a una singola parola. Jean-Baptiste Amadieu (B. examiné par le Saint-Office (1917-1927), pp. 13-18) prosegue il suo lavoro sulla censura ragionando sulle motivazioni che hanno spinto la Chiesa a non mettere all'Indice l'opera di B. e le ritrova nel fatto che il problema si pone quando è passato troppo tempo, e quando ormai troppi autori cattolici lo amano. JeanPaul Avice presenta tre note: la prima (Compléments iconographiques, pp. 19-24) rintraccia un autoritratto di Baudelaire nella sua riproduzione in incisione, e una fotografia nello studio di Carjat in cui spunta da una tenda. Nella seconda (L'homme, un Dieu qui a mal dîné), mostra che B. corregge la citazione da Hegel, ripresa da Saint-René Taillandier e Pierre Leroux, cambiando "bien dîné" in "mal dîné". Nella terza, Sur le "grand désert d'hommes" du "Peintre de la vie moderne" ripercorre la fortuna dell'espressione «vaste désert d'hommes» del René di Chateaubriand ripresa, prima ancora che da Baudelaire, da Stendhal e George Sand. Aurélia Cervoni (B. à Châteauroux. Un nouveau document, pp. 35-42) fa luce sugli avvenimenti della settimana in cui B. 
diventa caporedattore, à Châteauroux, del giornale conservatore "Le Représentant de l'Indre", il cui primo numero esce nell'ottobre 1848, riportando documentazioni circa un articolo scritto da B. per un giornale antagonista: articolo peraltro di cui non si trova traccia. L'A. pubblica in annesso l'articolo apparso su "La Meuse" con questa versione dell'episodio. Claire Chagniot (B. après 1862: comment oublier l'affront? pp. 43-50) esamina i rapporti di B. con l'incisore Bracquemond, di cui B. non aveva apprezzato il progetto di frontespizio per la seconda edizione delle Fleurs, e indaga sulle relazioni tra i due dopo il 1862. Antoine Compagnon (Ceux-là qui se bat... pour la Galette/C'est pas celuilà qui la mange, /il attrape les bons coups e pis c'est bon!, pp. 51-54) ricorda il giudizio negativo espresso da B. nei confronti del caricaturista Charlet, in contrasto con quello di Delacroix e di Daumier, e cita una caricatura di Daumier, in cui due straccivendoli commentano una litografia di Charlet (che dà il titolo all'articolo, e ha per oggetto appunto due ragazzi litigano per un tozzo di pane). Nello stesso tempo mette in evidenza come anche B. sia debitore del topos, nel poème en prose Le Gâteau. Sempre Compagnon (Ubi Troia fuit, pp. 57-59) rilegge il rapporto tra Jules Janin e B., che rimprovera al giornalista la perdita dell'ironia e l'ossequio al potere. Eppure il Gâteau des rois del Janin della prima ora ha influenzato non solo Le Spleen de Paris, ma anche, in maniera molto sottile e sotterranea, Le Cygne. Catherine Delons presenta l'unica lettera conosciuta di Jeanne Duval (una richiesta di denaro) oltre a quelle citate a memoria da Nadar; con Andrea Schellino propone invece alcune riflessioni a margine di una fotografia battuta all'asta nel novembre 2011, di cui è contestata, con acutezza di indagine, l'identità di Félicité Baudelaire, cognata del poeta. Jessica Desclaux (Un exemplaire des "Fleurs du mal" annoté par Barrès, pp. 73-82) rintraccia, nell'edizione delle Euvres complètes di B. regalate da Stanislas de Guaita a Barrès, i commenti a latere dei due autori, mostrando come i commenti di Barrès costituiscano una forma di autoriflessione. Wolfgang Drost (Baudelaire sympathisant du vitalisme, pp. 83-87) riflette sul termine "calorique", usato da Lavoisier, e impiegato da Baudelaire nel Salon del 1846 a indicare una natura esuberante, per mostrare ancora una volta la convinzione baudelairiana di una modernità legata all'esaurimento del vitalismo. Jacques Dupont (Baudelaire "probabiliste", pp. 89-94) si sofferma invece sul termine "probabiliste", insolito e invece legato alle teorie di Laplace, attraverso l'opera di Poe che aveva letto Laplace in francese, durante il soggiorno a West point nel 1830-31. Jean-Paul Goujon (Miettes de légendes, pp. 95-105) raccoglie una serie di battute di spirito attribuite a Baudelaire nel corso del tempo; André Guyaux (Des «Habitantes de l'île de Lesbos»?, pp. 107-114) ritorna sulla questione del valore semantico del termine Lesbiennes, ipotizzato da Baudelaire tra i primi titoli delle Fleurs, e ne ripercorre anche la successione degli annunci di pubblicazione, in alternanza con il titolo Le Catéchisme de la femme aimée. Appare anche l'incertezza del nome che B. vuole darsi: Baudelaire-Dufays o Dufaÿs, Pierre De Fayis, Charles Dufays. Lois Cassandra Hamrick (Eugénie Gautier, la "femme qui peint comme un homme», pp. 115-123) sbozza il ritratto della pittrice Eugénie Gautier, elogiata da B. - e non solo da lui - per un tipo di pennellata non femminile. Michele Hannoosh («Le Mauvais Vitrier» devant la justice, pp. 125-129) arricchisce l'interpretazione del poème en prose con la sfumatura del riferimento al processo contro le Fleurs, per cui l'accanimento contro il Vitrier è lo stesso, con le medesime accuse, di cui è stato oggetto il poeta. Jean-Marc Hovasse (B. et «Les Travailleurs de la mer», pp. 131-140) si curva sugli ultimi foglietti scritti da B. prima della paralisi, in cui B. prende annotazioni per una recensione all'opera di Hugo, e si sofferma sul valore da dare al termine "flatteur" utilizzato da B., la cui ambiguità è in contrasto con l'aspetto 
positivo delle note. Jean-Claude Larrat (Un cas de fraternité baudelairienne: Malraux, pp. 155-163) coglie le affinità tra i due autori nell'avversione per la natura e l'amore per l'artificio; ma al di là di quest'aspetto, che accomunerebbe anche i decadenti, sottolinea l'attenzione accordata da Malraux alla qualità di critico d'arte di B. e alla sua abolizione del racconto in poesia, che l'A. mette in confronto con le infrazioni alla continuità narrativa dei romanzi di Malraux. Yann Mortelette (Des Esseintes lecteur du "Spleen de Paris”, pp. 165-171) presenta la lettura dello Spleen da parte di Des Esseintes, che lo apprezza per il gusto della condensazione: condensazione che ha consentito amplificazioni successive, e non solo da parte dello stesso B.; tuttavia l'A. vede all'opera nello Spleen de Paris entrambe le poetiche. Lyne Penet (Sur un "oreiller de chair fraîche..." B. et Théophile Silvestre devant Rubens, pp. 175-180) mostra i debiti reciproci dei due autori nelle rispettive critiche d'arte, fino al momento in cui, dopo i loro viaggi in Belgio, Silvestre mostra entusiasmo per Rubens, mentre B. arriva a detestarlo. Samuel Rodary (B. au chat, pp. 181-185), cercando nelle carte di Champfleury, mette a confronto tre ritratti di B., di cui l'ultimo tratto dalla foto di Carjat del 1866. Andrea Schellino (Une lettre de B. à Charles Asselineau [?], pp. 189-194) presenta una lettera esclusa anche da Pichois, che pure ne fa menzione, perché troppo "grivoise" e che descrive un episodio divertente dove B. diventa vittima di un'incresciosa situazione per colpa di Boyer. JeanLuc Steinmetz (Dernières paroles, pp. 195-199) riprende un testo di B. dedicato al vino e all'haschich uscito sul "Messager de l'Assemblée" nel marzo 1851, mettendo in evidenza la frase messa in bocca a un ubriaco dove compaiono di seguito "tête, armée" e ne ricostruisce il percorso filologico, da Les derniers moments de Napoléon riportati dal medico, a Nerval, che le utilizza come titolo di un sonetto rimasto inedito e in una poesia di giovinezza, fino a Dumas nel suo Napoléon (1840). Jérôme Thélot (Sur le sadisme de B, de Georges Blin, pp. 203-208) riprende il saggio scritto da Blin contro Sartre, dove Blin dimostra che, se il sadismo come natura umana accomuna B. e Sartre, e se l'atto sessuale può essere inteso come atto di aggressività, tuttavia per Sade l'altro non esiste; mentre l'attenzione piena di carità per l'altro di B. arriva a fargli trascurare l'io. L'A. osserva però come Blin abbia trascurato il fatto che Sade scrive in prosa e B. in poesia, e quindi per B. l'immagine sia altrettanto importante della natura: nel suo caso perciò il sadismo ha costituto un elemento di esaltazione delle immagini. Eduardo Horta Nassif Vera (Entre poncif et théologie: le lieu commun dans la poésie de B., pp. 209-215), in una mise en abyme filologica, prende le mosse da un intervento di Patrick Labarthe pubblicato nella prima «Année baudelairienne», per ricavarvi la similitudine tra lo spazio delle strade parigine e quello del linguaggio. Isola quindi i termini chaos e tohu-bohu e ne constata la variazione nell'accezione, per una diminuzione della carica simbolica fino al luogo comune. Con questo l'A. arriva perciò a dimostrare il raggiungimento dell'equivalenza simbolica tra vuoto esterno e vuoto del linguaggio. Alexandre de Vitry (Le lexique baudelairien de la fraternité, pp. 217-221) mostra l'evoluzione dell'accezione del termine in B., dalla rivoluzione del ' 48 in cui si accorda con la retorica rivoluzionaria, per ironizzare in seguito proprio sulla fraternizzazione e concepire alla fine solo una fraternità di spiriti attraverso le opere, lungo le epoche. Fabrice Wilhelm (Théo Wagner illustrateur des "Fleurs du mal", pp. 222-233) mette l'accento su di un illustratore quasi cancellato dal tempo, dal momento che la maggior parte delle opere sono andate distrutte. Modello di nudo per i pittori, acrobata, trapezista, amico di Salis e habitué dello Chat noir della prima ora, adepto dei Rose+Croix catholiques prima della scissione voluta da Péladan, e, soprattutto, amico dei fratelli La Rochefoucauld, aveva immaginato un'edizione illustrata delle Fleurs di cui ci restano poche planches. Julien 
Zanetta (Un dialogue au Purgatoire? B. et Poe selon Fantin-Latour, pp. 234-239) studia un disegno di Fantin-Latour, di due figure sotto alle quali l'artista ha messo il nome di B. e Poe e riconosce nell'inquadratura un richiamo a Dante e Virgilio nella Divina commedia, con Poe che - forse in quanto autore di Eureka - si mostra come una figura di rivelazione. 